\title{
Low-temperature phonon damping in vitreous silica explored by UV Brillouin spectroscopy
}

\author{
G. BALDI*†ף, S. CAPONI $\uparrow$, A. FONTANA $\uparrow$, P. BENASSI $\uparrow$,

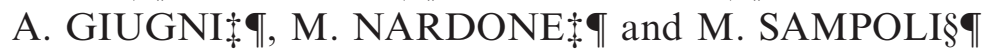 \\ $\dagger$ Department of Physics, University of Trento, Via Sommarive 14, \\ 38050 Povo, Trento, Italy \\ tDepartment of Physics, University of L'Aquila, Via Vetoio, Coppito, \\ 67100, L'Aquila, Italy \\ $\S$ Department of Energetic, University of Firenze, 50139, \\ Firenze, Italy \\ 『CRS SOFT-INFM-CNR, c/o University of Roma La Sapienza, \\ 00185 Roma, Italy
}

(Received 28 May 2006; in final form 20 June 2006)

\begin{abstract}
The recently developed technique of Brillouin ultraviolet scattering is used to measure phonon dispersion and damping in the prototypical strong glass $\mathrm{v}-\mathrm{SiO}_{2}$. Results on the low temperature $(T \sim 10-100 \mathrm{~K})$ sound attenuation at a frequency of $\sim 66 \mathrm{GHz}$ are presented. The comparison between a model, which takes into account dynamical processes (thermally activated relaxations and the interaction with the bath of thermal vibrations), and the experimental data indicates the presence of a strong static contribution in the region explored by ultraviolet radiation, supporting the presence of a transition between static and dynamic attenuation mechanisms at a frequency of $\sim 100 \mathrm{GHz}$.
\end{abstract}

\section{Introduction}

The study of the low-energy vibrational dynamics in glasses is one of the most intriguing challenges in understanding the effects of topological disorder in solids. Ultrasonic measurements (see [1] and references therein) at low frequency $(v<1 \mathrm{GHz})$ and Brillouin scattering [2] at higher frequencies $(v>1 \mathrm{GHz})$ allow experimental determination of the velocity and damping of collective excitations in glasses. Thus, these techniques are the most accurate for studying the low-energy tail $(v<1 \mathrm{THz})$ of the vibrational spectrum in amorphous systems.

Determination of the frequency and temperature dependence of sound attenuation is important in understanding the nature of low-frequency excitations in glasses. This particular frequency region, lying at and below the Boson peak, is practically inaccessible to numerical simulations via realistic classical systems $[3,4]$ and, thus, the nature of the corresponding eigenmodes is still uncertain. Various studies $[5,6]$ have suggested that both perturbed plane waves, following the

*Corresponding author. Email: baldi@science.unitn.it 
correct scheme of crystal acoustic modes and some quasi-localized modes, associated with small values of the participation ratio, could coexist at and below the Boson peak frequency. Moreover, the mean free path of acoustic waves is strictly related to transport properties of the system, such as thermal conductivity. Temperature dependence is one of the unsolved problems in the physics of structural glasses, despite the large number of studies on the subject (see, for example, [7] and [8]).

In ultrasonic measurements (US), as well as in Brillouin light-scattering (BLS), sound attenuation is strongly temperature-dependent, showing, almost universally, a broad maximum below the glass transition temperature. The position of the maximum increases smoothly and the amplitude increases almost linearly with increasing frequency. Such behaviour is generally understood in terms of a thermally activated relaxation process with the relaxation time following an Arrhenius-type activation law [9].

Vitreous silica, considered the prototype of strong glasses and a model for all glasses [10] is the most widely studied. In particular, sound attenuation has been the subject of a large number of experimental studies using various techniques [11-16]. In $\mathrm{v}-\mathrm{SiO}_{2}$ at ultrasonic frequencies $(\mathrm{MHz})$, attenuation, as a function of temperature, shows a well-defined peak, while at hypersonic frequencies $(\mathrm{GHz})$, this temperature dependence, although still characterized by a maximum located around $150 \mathrm{~K}$, clearly shows a plateau at higher temperatures. Wavevector dependence of attenuation at room temperature in the region of frequency explored by BLS and Brillouin ultraviolet light scattering (BUVS) has been recently observed [16] to scale with the square of the frequency and, thus, with the square of the wavevector, the dispersion curve being linear in this region. A $q^{2}$ dependence of attenuation was also observed at much higher frequencies $(v=1-10 \mathrm{THz})$ by means of inelastic X-rays scattering (IXS). Measurements of sound attenuation by IXS showed the absence of any temperature dependence in the explored temperature range [17], thus, suggesting a static nature for attenuation in this region.

Recent BUVS room-temperature measurements [18] suggest that the transition from the dynamic to the static attenuation regime should occur at around $100 \mathrm{GHz}$. The presence of such a crossover should be directly revealed in the temperature dependence of sound attenuation, which should decrease progressively from the frequency region explored by BLS to the crossover frequency.

In this paper, we present measurements of sound attenuation in vitreous silica at low temperatures, using the high-resolution ultra-violet spectrometer (HIRESUV) at the University of L'Aquila [19]. The frequency and damping of longitudinal phonons of $\mathrm{v}-S i O_{2}$ at the wavevector $q=0.071 \mathrm{~nm}^{-1}$ have been measured in a temperature range from $\sim 10$ to $100 \mathrm{~K}$. Experimental results for acoustic attenuation are compared with predictions of a phenomenological model, which takes into account not only the relevant dynamical mechanisms in the temperature range, namely the thermally activated relaxation processes [20], but also the interaction with thermal vibrations [21]. The aim is to show how a comparison between expected behaviour and measured sound attenuation indicates the presence of a relevant static contribution in ultraviolet frequencies, which is almost negligible in the region explored by BLS. 


\section{Experiment and results}

The newly developed technique of Brillouin ultraviolet light scattering (BUVS) is used to explore the frequency region between that accessible by BLS and IXS. The HIRESUV spectrometer is used with excitation of $266 \mathrm{~nm}$ and at near-backscattering configuration, a $173^{\circ}$ scattering angle, in $\mathrm{v}-\mathrm{SiO}_{2}$, corresponding to $q=0.071 \mathrm{~nm}^{-1}$. The instrument is based on a double $f 1: 20$ laboratory-made monochromator of $4 \mathrm{~m}$ focal length specifically designed for Brillouin spectroscopy with both visible $(532 \mathrm{~nm})$ and ultraviolet $(266 \mathrm{~nm})$ excitations; a detailed description of the instrument can be found in [19]. The experiment was performed on a spectrosil sample purchased from SILO (Firenze, Italy). The sample is characterized by an $\mathrm{OH}^{-}$content of $1500 \mathrm{ppm}$, determined by means of infrared absorption, and consistent with literature values for spectrosil samples [22].

Longitudinal and transverse sound velocities, $v_{L, T}$, were determined from the measured frequency shifts $v_{L}$ and $v_{T}$ by the following formula:

$$
v_{L, T}=\frac{2 \pi v_{L, T}}{q}
$$

where $q$ is the exchanged wavevector fixed by the experimental geometry and wavelength of the incident radiation. Damping of the acoustic waves is obtained from the spectral width, full-width half-maximum (F.W.H.M.; $\Gamma$ ) of the Brillouin lines. Assuming that the isotropic contribution to the light scattering signal dominates the spectrum, the spectra are proportional to the dynamic structure factor, $S(q, v)$, convoluted to the resolution function $R(v)$, according to the standard theory of Brillouin scattering in glasses [23]. The dynamic structure factor, derived from a memory function approach to the dynamics of density fluctuations in the Markov approximation, can be described as a damped harmonic oscillator (DHO) for the inelastic contribution and a delta function for the elastic scattering [24].

In the region of frequencies below $10 \mathrm{GHz}$, the peak of attenuation is found to scale almost linearly with frequency, so that the internal friction, $Q^{-1}=\Gamma / v$, is the natural quantity to deal with. Moreover, it is a dimensionless quantity and has an immediate physical meaning; for example, $Q^{-1} \approx 1$ defines the Ioffe-Regel regime (different definitions of this limit can be found in the literature [25], such as $Q^{-1} \approx 1$ / $\pi$ or $Q^{-1} \approx 1 / 2 \pi$ ), where attenuation is comparable to frequency.

An example of a spectrum, Stokes line, obtained at $T \sim 10 \mathrm{~K}$ is shown in figure 1 together with the best-fitting function and the scaled elastic line used as resolution. Particular attention has been adopted during the fitting procedure to take into account the weak residual signal due to scattering from the $\mathrm{v}-\mathrm{SiO}_{2}$ windows of the cryostat itself - an important term in lowering the temperature of the sample. This term appears as a small bump on the high frequency side of the Brillouin peak. The extremely high contrast of the spectrometer (up to $10^{10}$ ) may be appreciated in the completely flat background, which characterizes the spectrum. The variation in sound velocity $v_{L}$ as a function of temperature is shown in figure 2 , and is in good agreement, within experimental error, with previous visible and ultrasonic data. Small differences with the ultrasonic data [9] may be associated with the different thermal histories of the silica samples. 


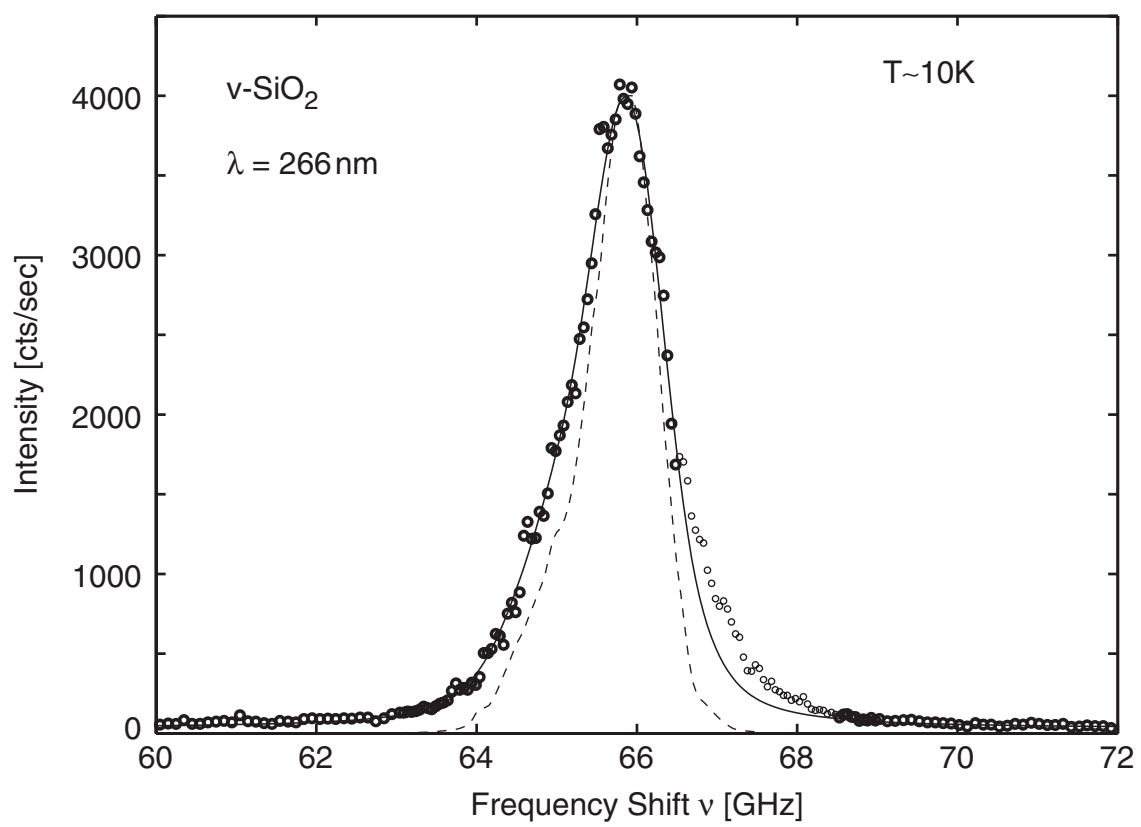

Figure 1. Brillouin spectrum (Stokes side) of vitreous silica at $T=(10 \pm 3) \mathrm{K}$ in nearbackscattering configuration obtained with a laser source operating at $\lambda=266 \mathrm{~nm}$. The exchanged wavevector is $q=0.071 \mathrm{~nm}^{-1}$. The continuous line superimposed to the experimental data (o) is the best-fitted line shape; it is obtained considering only the marked circles, as described in the text. The dashed line aligned with the Brillouin peak is the instrumental resolution function. Resolution (F.W.H.M.) $=1.1 \mathrm{GHz}$, deconvolved line width (F.W.H.M.) $=0.45 \mathrm{GHz}$.

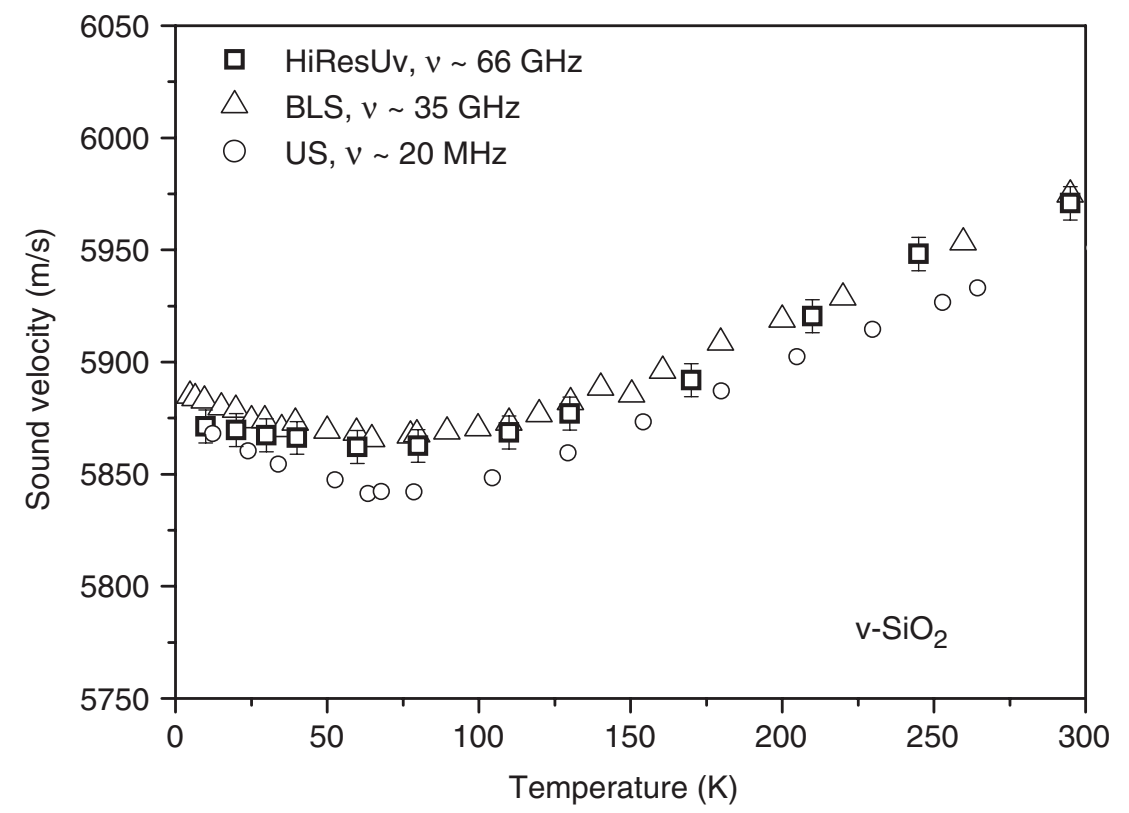

Figure 2. Temperature dependence of the sound velocity in vitreous silica as measured by BUVS (squares: present work), BLS (triangles: [12]) and US (circles: [9]). 
In figure 3, the internal friction, $Q^{-1}$, calculated from the BUVS data, together with visible measurements from [12] and ultraviolet data from the IUVS beam line in Trieste [15], are reported. The difference between the visible and ultraviolet data is evident and supports the idea that, in the frequency regions explored by ultraviolet excitation, another attenuation mechanism is added to the dynamical contribution.

The temperature dependence of the internal friction corresponding to five ultrasonic frequencies $(11.4,180 \mathrm{kHz}, 21,207$ and $748 \mathrm{MHz})$ is shown in figure 4, together with the BLS measurement. The plot shows that the attenuation peak varies by a factor five in a window of more than six decades in frequency, i.e. $Q^{-1}$ is almost constant up to the region investigated by BLS.

\section{Discussion}

It is well established that sound attenuation in the US region $(<1 \mathrm{GHz})$ in vitreous silica is dominated by thermally activated relaxation processes (TAR), although their microscopic origin is still debated. We have analyzed US data using the following model for the TAR contribution (see [26] and references therein):

$$
Q_{T A R}^{-1}(\omega, T)=C \Phi\left(\frac{\sqrt{2} T}{\sigma_{\Delta}}\right) \frac{1}{T} \int_{0}^{\infty}\left(\frac{V}{\sigma_{V}}\right)^{-\zeta} \exp \left(-\frac{V^{2}}{2 \sigma_{V}^{2}}\right) \frac{\omega \tau_{0} \exp (V / T)}{1+\omega^{2} \tau_{0}^{2} \exp (2 V / T)} d V
$$

where $\Phi(x)$ is the error function and $\omega=2 \pi v$.

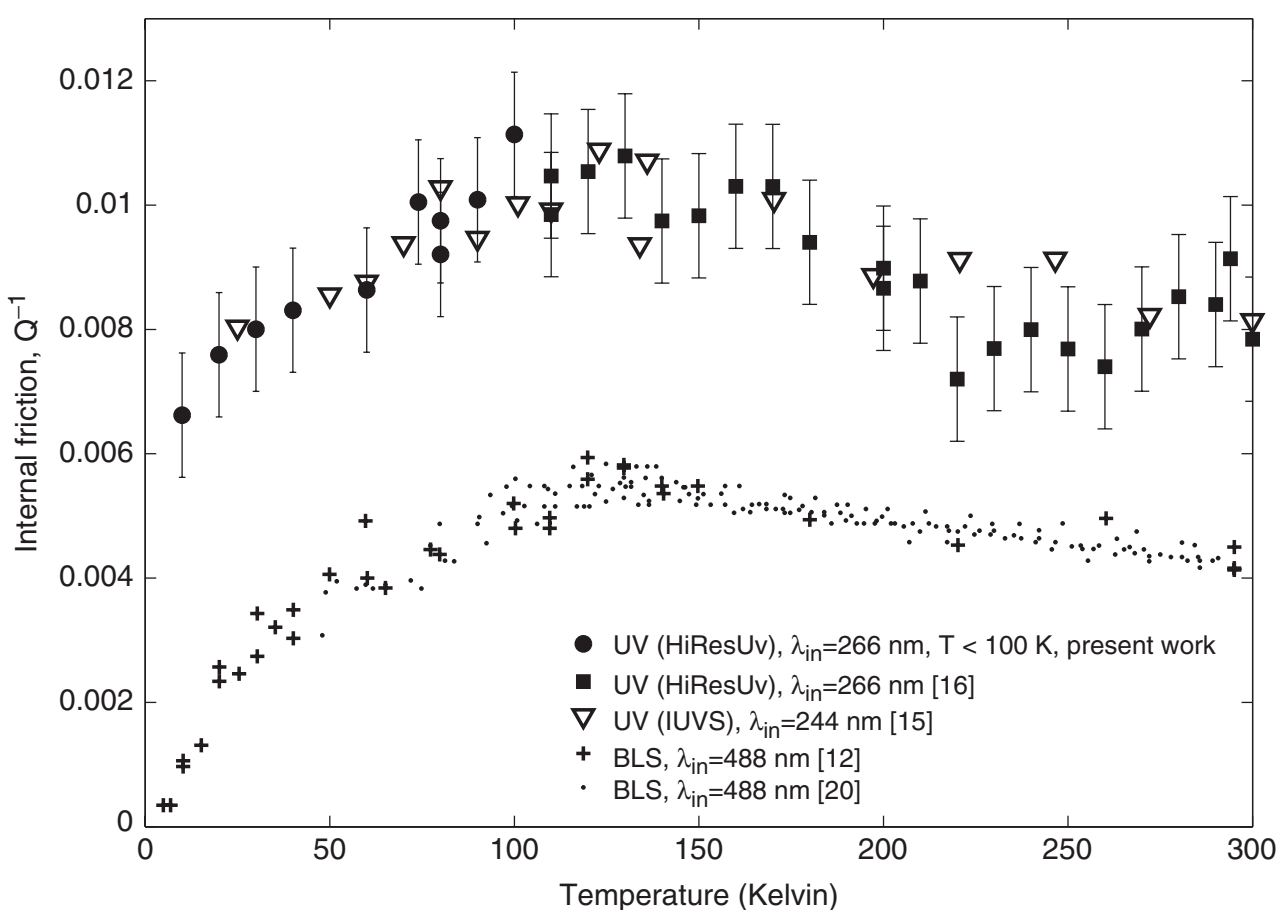

Figure 3. Temperature dependence of the internal friction $\left(Q^{-1}=\Gamma / v\right)$ in vitreous silica as measured with IUVS (triangles: [15]), HiResUV (filled circles: present work; filled squares: [16]), BLS (crosses: [26]; dots: [20]). 


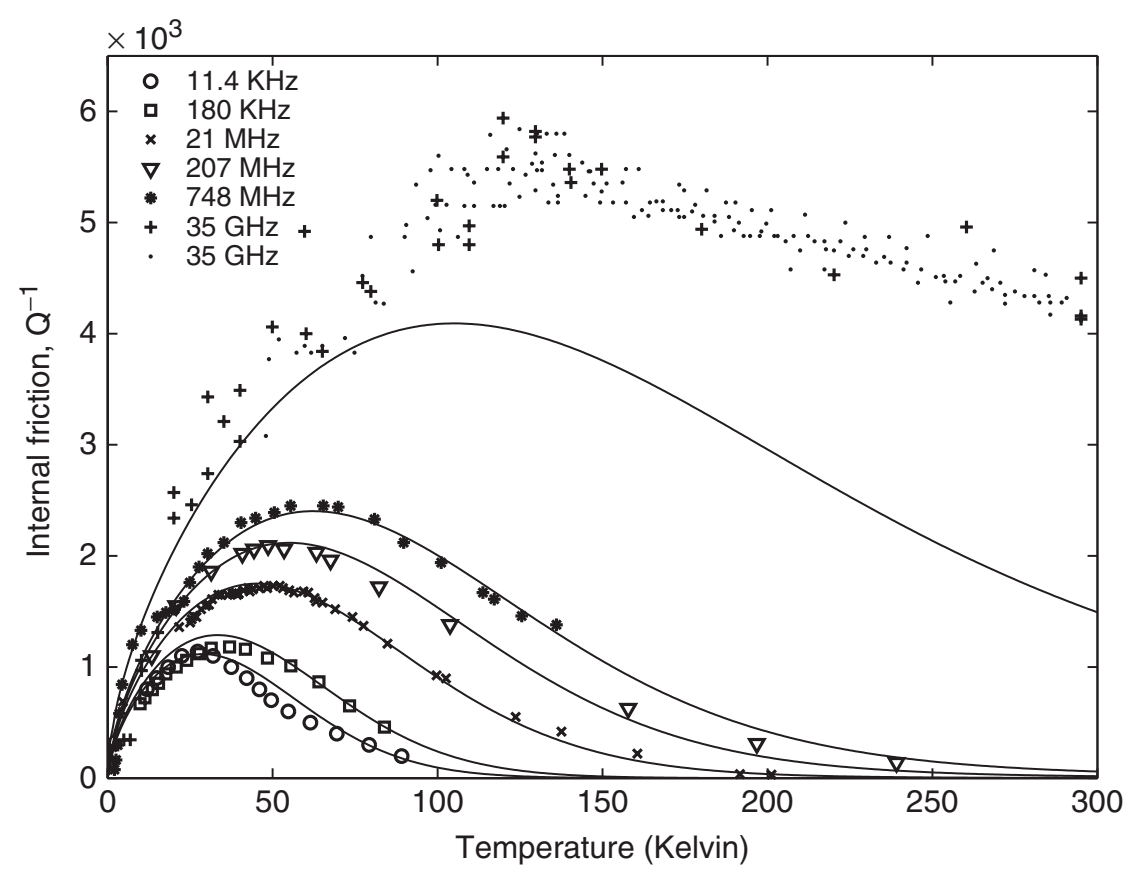

Figure 4. Temperature dependence of the internal friction in vitreous silica at different fixed frequencies. Circles: US, $11.4 \mathrm{kHz}$ [20]; squares: US, $180 \mathrm{kHz}$ [27]; products: US, $21 \mathrm{MHz}$ [28]; triangles: US, $207 \mathrm{MHz}$ [12]; stars: US, $748 \mathrm{MHz}$ [29]; crosses [26] and points [20]: BLS, $35 \mathrm{GHz}$. The continuous lines are the best fitted functions to the five US datasets with the model of equation (2) with the parameter $\sigma_{\Delta}$ fixed at $10^{4} \mathrm{~K}$. Parameters resulting from the fit: $\tau_{0}=1.3 \cdot 10^{-14} \mathrm{~s}, \sigma_{V}=770 \mathrm{~K}, \zeta=0.039$ and $C=0.19$.

Five parameters are entered into the model of equation (2): minimum relaxation time, $\tau_{0}$; two parameters describing the distribution of activation energies, $\sigma_{V}$ and $\zeta$; the variance of asymmetry distributions in the double well potentials, $\sigma_{\Delta}$; and a multiplicative constant $C$. Assuming a uniform distribution of asymmetries (see [20] and references therein), the parameter $\sigma_{\Delta}$ was fixed at a value much greater than $\sigma_{V}$, i.e. $\sigma_{\Delta}=10^{4} \mathrm{~K}$. The remaining four parameters can be determined by fitting the temperature dependence of the measured $Q^{-1}$ at a given frequency. A more accurate estimate of the parameters can be obtained by a simultaneous fit of the data at different frequencies.

From the fitting procedure to the five US datasets presented in figure 4, with $\sigma_{\Delta}$ fixed at $10^{4} \mathrm{~K}$, one obtains the following set of parameters: $\tau_{0}=1.3 \cdot 10^{-14} \mathrm{~s}$, $\sigma_{V}=770 \mathrm{~K}, \zeta=0.39$ and $C$ variable between 0.17 and 0.19 . The result of the fitting procedure on the five sets of US measurements and extrapolation to the BLS, $v=35 \mathrm{GHz}$, data is shown in figure 4 . In the plot, the multiplicative constant $C$ has been fixed at 0.19 for all the curves. The remarkably good agreement between the model and the ultrasonic measurements can be appreciated, both for the position of the attenuation peak, which is well reproduced in all US curves, and also for the intensities, although small discrepancies can be seen. The five US frequencies have been chosen because they are almost linearly displaced in a logarithmic scale and for the good quality of the data. These sets of data have been obtained in the past by 
various authors $[12,20,27-29]$ with slightly different ultrasonic techniques and with different samples, so that a certain discrepancy between the model and the data is not surprising.

The good agreement between the model and the US data demonstrates that a uniform distribution of asymmetries in the double wells can be assumed. With this assumption, the model can reproduce the position of the peak in the BLS data but still underestimates the internal friction at this frequency; in particular, it does not describe its high-temperature tail. The discrepancy between the TAR model and the BLS data can be linked to the anharmonic contribution, $Q_{A N H}^{-1}$, arising from the interaction between the acoustic wave and the bath of thermal phonons. It is natural that this term is stronger at higher temperatures, where the amplitude of atomic displacements is higher.

A detailed description of the anharmonic contribution, in the Akhiezer formulation, would require numerical treatment, as discussed in [21]. Unfortunately, a numerical approach suffers from the limited dimensions of the sample that can be modelled on a computer. This difficulty would affect, in particular, the contribution from the low frequency tail of the thermal phonon distribution. In the absence of a more general theory describing the anharmonicities, a tentative description of this contribution to sound attenuation is obtained by assuming that the effect of the bath of thermal phonons on the acoustic wave can be described by a single relaxation time $\tau_{t h}(T)$, which depends on temperature. The anharmonic contribution to internal friction can then be modelled as [2]:

$$
Q_{A N H}^{-1}(\omega, T)=A T \frac{\omega \tau_{t h}(T)}{1+\omega^{2} \tau_{t h}^{2}(T)}
$$

The value of thermal relaxation time $\tau_{t h}(T)$ and its temperature dependence have to be determined from the experimental data. At a fixed frequency $\Omega$, the anharmonic term of equation (3), divided by temperature, peaks at that temperature $T_{m}$ for which $\Omega \tau_{t h}\left(T_{m}\right) \sim 1$. If this temperature lies in the experimental region, it is possible to determine $\tau_{t h}(T)$ by comparing equation (3) to the experimental data, because coefficient $A$ can then be fixed by the value of the peak: $Q_{A N H}^{-1}\left(\Omega, T_{m}\right) / T_{m}$.

The anharmonic contribution, at a given frequency, can be estimated by subtracting the TAR contribution, at that frequency, from the experimental data. A small static contribution is also subtracted, as suggested by the recent observation of a crossover between static and dynamic contributions at a frequency around $100 \mathrm{GHz}$ [18]. The value of such a static contribution in the explored BLS region $(v \sim 35 \mathrm{GHz})$ can be estimated from the minimum of $Q^{-1}$, which is observed at temperatures around $5 \mathrm{~K}$ (with $\sigma_{\Delta}$ fixed at $10^{4} \mathrm{~K}$ ).

Once the anharmonic contribution, $Q_{A N H}^{-1}$, has been determined, the thermal relaxation time $\tau_{t h}$, in the temperature range of the experimental data, can be estimated by solving equation (3), as suggested in [26]. Fitting the temperature dependence of the thermal relaxation time with an appropriate function, one obtains a model for the anharmonic term of equation (3) and frequency and temperature dependence can be predicted. Temperature dependence of the thermal relaxation 
time, $\tau_{t h}$, is, however, affected by strong fluctuations at low temperatures. For this reason, a simple linear fit on the logarithmic scale is chosen: $\ln \tau_{t h}(T)=a+b \ln T$, with $a=-20.8$ and $b=-1.0$. With this description of the thermal relaxation time, BLS data are described, as shown by the continuous line in figure 5 . In the same figure, extrapolations of the TAR contribution of equation (2) and of the anharmonic term (3) at $v \sim 66 \mathrm{GHz}$ are also presented as a dashed line. It appears that the dynamical terms, $Q_{T A R}^{-1}+Q_{A N H}^{-1}$, can account only for half of the sound attenuation measured with the HIRESUV spectrometer.

On the other hand, if a static contribution $Q_{S}^{-1}=0.004$ is added to the dynamical terms, then qualitatively, good agreement between UV data and the model can be appreciated, as shown by the dash-dotted line in figure 5. This value for the static term, introduced to describe the UV data, is in qualitative agreement with the results of recent measurements around $100 \mathrm{GHz}$ [18].

It should, however, be noted that the $\mathrm{OH}^{-}$content may have a significant influence on the shape and intensity of the attenuation peak found in temperature dependence around $150 \mathrm{~K}$, as pointed out in a recent paper [22]. Nevertheless, the $\mathrm{OH}^{-}$content does not affect the attenuation for temperatures outside the peak region, below 50 and above $250 \mathrm{~K}$, where the discrepancy between our UV measurement and the model (dashed line of figure 5) is evident.

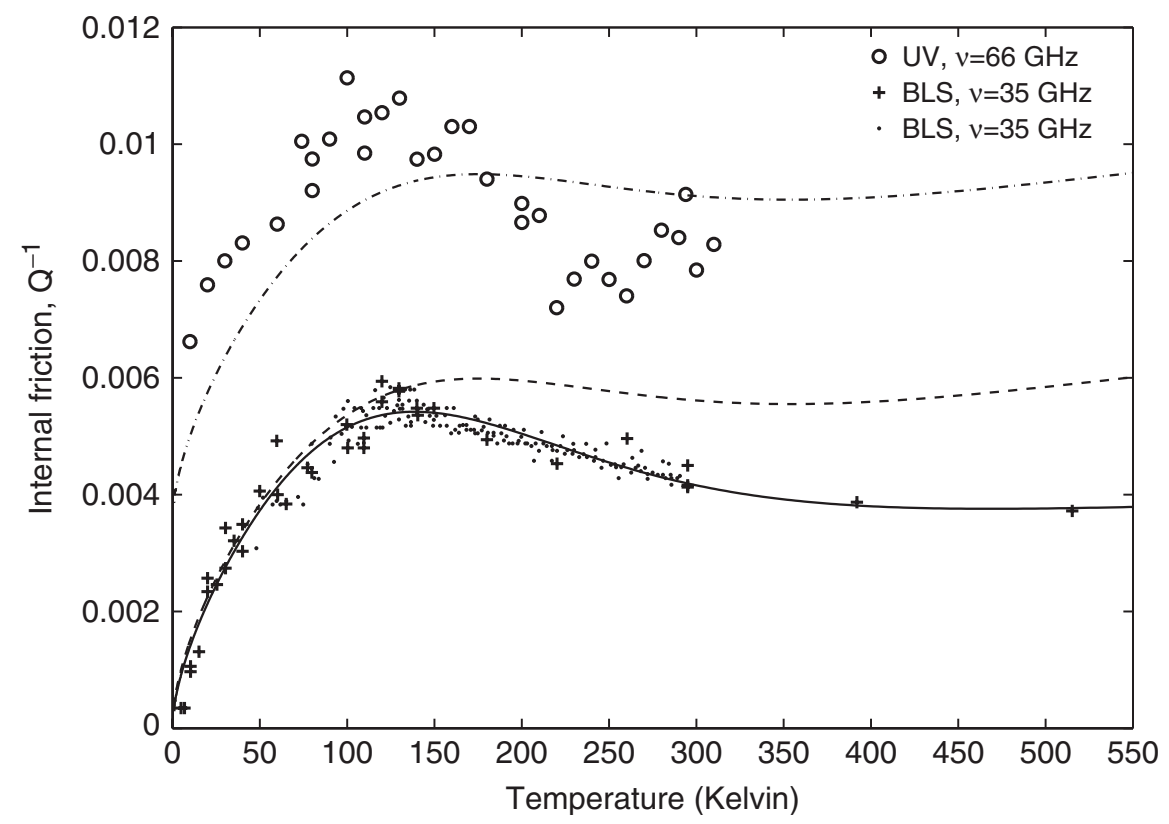

Figure 5. Internal friction in vitreous silica at $v=35 \mathrm{GHz}$, crosses [26] and points [20] and $v=66 \mathrm{GHz}$, circles (present work and [16]). The continuous line is obtained using equations (2) and (3) at $35 \mathrm{GHz}$, as discussed in the text. The dashed line is the extrapolation of the sum of equations (2) and (3) to the ultraviolet regime, $v=66 \mathrm{GHz}$. The dash-dotted line is obtained by summing to the dashed curve a static, temperature-independent, contribution $Q_{S}^{-1}=0.004$. 


\section{Conclusions}

We presented new Brillouin ultraviolet light scattering data at the low temperature range in $\mathrm{v}-\mathrm{SiO}_{2}$. We compared the data to a model accounting for both thermally activated relaxations and the interaction with the bath of thermal vibrations. The extension of the model to the ultraviolet region shows that dynamical, or anharmonic, contributions can account for only half of the observed internal friction, supporting the idea of the existence of a strong static contribution in the region explored by ultraviolet spectroscopy.

If the static term is included, as in the dash-dotted line of figure 5, the resulting description is in qualitative agreement with the ultraviolet $(66 \mathrm{GHz})$ data, which are affected by a relative error of $\sim 20 \%$. This supports the description of the Akhiezer mechanism in terms of equation (3) with a single thermal relaxation time. Further testing of equation (3) may be performed, for example, by measuring the temperature dependence of sound attenuation at different scattering angles between the visible and ultraviolet backscattering exchanged wavevectors.

To investigate the nature of this new attenuation term, further measurements at low temperature with the HIRESUV spectrometer are planned.

\section{References}

[1] S. Hunklingher and W. Arnold, in Physical Acoustics, edited by W.P. Mason and R.N. Thurston, Vol. XII (Academic Press, New York, 1976), p. 155.

[2] A.S. Pine, Phys. Rev. 185, 1187 (1969).

[3] R. Dell'Anna, G. Ruocco, M. Sampoli, et al., Phys. Rev. Lett. 801236 (1998).

[4] J.L. Feldman, P.B. Allen and S.R. Bickham, Phys. Rev. B 593551 (1999).

[5] U. Buchenau, Yu.M. Galperin, V.L. Gurevich, et al., Phys. Rev. B 462798 (1992).

[6] S.N. Taraskin and S.R. Elliott, Phys. Rev. B 598572 (1999).

[7] M.S. Love and A.C. Anderson, Phys. Rev. B 421845 (1990).

[8] P.B. Allen and J.L. Feldman, Phys. Rev. B 4812581 (1993).

[9] S. Hunklinger and M. Schifus, in Amorphous Solids: Low Temperature Properties, Topics in Current Physics, edited by W.A. Phillips, Vol. 24 (Springer, Berlin, 1981), p. 81.

[10] C.A. Angell, Science 2671924 (1995).

[11] W. Dietsche and H. Kinder, Phys. Rev. Lett. 431413 (1979).

[12] R. Vacher, J. Pelous, F. Plicque, et al., J. Non-Cryst. Solids 45397 (1981).

[13] T.C. Zhu, H.J. Maris and J. Tauc, Phys. Rev. B 444281 (1991).

[14] P. Benassi, M. Krisch, C. Masciovecchio, et al., Phys. Rev. Lett. 773835 (1996).

[15] C. Masciovecchio, A. Gessini, S. Di Fonzo, et al., Phys. Rev. Lett. 92247401 (2004).

[16] P. Benassi, S. Caponi, R. Eramo, et al., Phys. Rev. B 71172201 (2005).

[17] G. Ruocco, F. Sette, R. Di Leonardo, et al., Phys. Rev. Lett. 835583 (1999).

[18] C. Masciovecchio, G. Baldi, S. Caponi, et al., Phys. Rev. Lett. (in press).

[19] P. Benassi, R. Eramo, A. Giugni, et al., Rev. Sci. Instrum. 76013904 (2005).

[20] D. Tielburger, R. Merz, R. Ehrenfels, et al., Phys. Rev. B 452750 (1992).

[21] J. Fabian and P.B. Allen, Phys. Rev. Lett. 821478 (1999).

[22] C. Levelut, R. Le Parc and J. Pelous, Phys. Rev.B 73052202 (2006).

[23] J.R. Sandercock, in Light Scattering in Solids III, Topics in Applied Physics, edited by M. Cardona and G. Guntherodt, Vol. 51 (Springer, Berlin, 1982), p. 173. 
[24] J.P. Boon and S. Yip, Molecular Hydrodynamics (Dover Press, New York, 1980).

[25] S.N. Taraskin and S.R. Elliott, Phys Rev. B 6112031 (2000).

[26] R. Vacher, E. Courtens and M. Foret, Phys. Rev. B 72214205 (2005).

[27] R. Keil, G. Kasper and S. Hunklinger, J. Non-Cryst. Solids 164/166 1183 (1993).

[28] R.E. Strakna and H.T. Savage, J. Appl. Phys. 351445 (1964).

[29] C.K. Jones, P.G. Klemens and J.A. Rayne, Phys. Lett. 831 (1964). 